\title{
Codeine Precipitating Serotonin Syndrome in a Patient in Therapy with Antidepressant and Triptan
}

\author{
Giulia Milano ${ }^{1}$, Werner Maria $\mathrm{Natta}^{2}$, Alfredo Bello ${ }^{2}$, Antonietta Martelli ${ }^{1}$, Francesca Mattioli ${ }^{1}$ \\ 'Department of Internal Medicine, Clinical Pharmacology and Toxicology Unit, University of Genoa, Genoa, ${ }^{2}$ Neurology Unit, Istituto Clinico 'Salus', \\ Alessandria, Italy
}

\begin{abstract}
The serotonin syndrome is a serioius medical condition due due to an intensive stimulation of setonin receptors. It is a rare, but severe, consequence of interaction between serotomimetic agents. This is a report of a 70-year-old woman steadily in therapy with venlafaxine and rizatriptan for migraine and major depressive syndrome. She was admitted to neurology unit for decreased light reflex with miotic pupils, global hyperreflexia, tremor, anxiety, ataxia and incoordination. The patient was diagnosed as a probable case of serotonin syndrome due to a pharmacological interaction between venlafaxine and rizatriptan trigged by opioid intake. In this paper, the development of syntomatology, the clinical examination and the possible pharmacokinetics explanation were carefully discussed and analysed.
\end{abstract}

KEY WORDS: Drug-drug interactions; Codeine; Serotonin syndrome; Prescription drug misuse; Migraine disorders; Major depressive disorder.

\section{INTRODUCTION}

Drug interactions are nowadays a very serious problem and they can frequently include common prescribed drugs and over-the-counter (OTC) medicines. ${ }^{1,2)}$ Antidepressants are very frequently prescribed drugs, they include selective serotonin reuptake inhibitors (SSRIs) and serotonin-noradrenaline reuptake inhibitors (SNRIs), prescribed for the treatment of major depressive disorder. ${ }^{3}$

The SSRIs mechanism of action is to increase the intra-synaptic level of seronin with the inhibition of serotonin reuptake. The same is true for SNRIs respect to noradrenaline. As a secondary mechanism of action, SSRIs increase efficacy of 5-hydroxytryptamine (5-HT) neurons by desensitizing 5-HT autoreceptors of the serotonin nerve terminals. ${ }^{4,5)}$

Adverse events (AEs) of SSRIs and SNRIs include: hyponatremia, risk of ischemic stroke and intracranial hemorrhages, ${ }^{6}$ suicidality and mania. ${ }^{7}$ The serotonin syn-

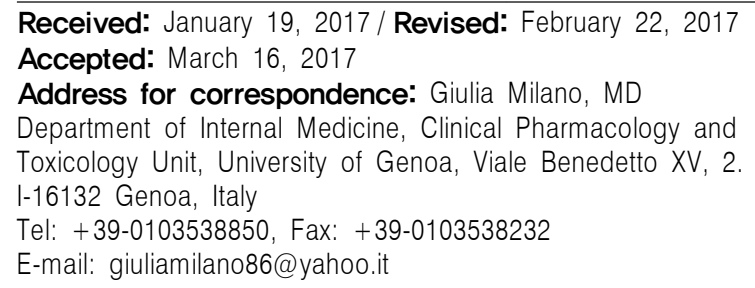

action; it is a rare consequence of interaction between serotomimetic agents. The SS is considered to be due to an intensive stimulation of $5-\mathrm{HT}_{1 \mathrm{~A}}$ (in central grey nuclei and in the medulla) and of 5- $\mathrm{HT}_{2 \mathrm{~A}}$ (in the central and peripheral nervous system) receptors by serotonergic medicines ${ }^{8}$; it is characterized by neuromuscular hyperactivity (myoclonus and hyperreflexia), cognitive alterations (agitation and confusion) and autonomic disorders (hyperthermia and tachycardia). ${ }^{9)}$ The SS is caused by the serotonergic agent's use and it usually occurs within 24 hours after the serotomimetic drugs intake. ${ }^{8-10)}$ Abadie et al. ${ }^{11)}$ described the mostly involved medicines in SS, based on the French pharmacovigilance reports; SSRIs seem to be involved in $42.1 \%$ and SNRIs (mainly venlafaxine) in $9.1 \%$.

Triptans (5-HT receptor agonists) are another drug category selectively acting on the serotonin receptors. Triptans are 5- $\mathrm{HT}_{1 \mathrm{~B} / 1 \mathrm{D}}$ receptor agonists with lower affinity also for $5-\mathrm{HT}_{1 \mathrm{~A}}$ and $5-\mathrm{HT}_{2 \mathrm{~A}}$ receptors, ${ }^{12)}$ they are used in migraine's therapy and they usually have dose-dependent side effects (paresthesia, nausea, fatigue, dry mouth and facial flushing). ${ }^{13,14)}$

We report a case of SS probably induced by interaction between one antidepressant and one triptan with codeine.

Codeine, 3-methylmorphine, is a natural methylated form of morphine. Codeine is metabolized into morphine 
(and its metabolites) and into codeine-6-glucuronide and norcodeine; the principal cytochromes involved in metabolic codeine pathway are CYP2D6 and CYP3A4. ${ }^{15)}$ Worldwide codeine, in combined preparation with acetaminophen, is the most commonly opiate prescribed used in the treatment of mild to moderate pain and as an antitussive medicine, indeed in different countries (i.e., United Kingdom, South Africa, Ireland, France, and Australia) is an OTC medicine. ${ }^{16-18)}$ The AEs of codeine are the same of those of opioid category (sedation, nausea, vomiting, constipation and respiratory depression). ${ }^{19)}$

The aim of this report is to describe an interesting and not yet reported AE observed in one patient steadily in therapy with venlafaxine and rizatriptan. We have analyzed the development of symptomatology after added therapy with codeine and the possible association with opioid treatment.

\section{CASE}

The patient is a 70-year-old woman, she has a long clinical history of migraine and major depressive syndrome. She describes tension-type headache and depressive syndrome with anxiety and insomnia since she was 20 years old.

During the last 10 years she was treated with individual support psychotherapy and she received several psychopharmacological therapies, such as valproate, topiramate, propranolol and amitryptiline (as a long term therapy), acetaminophen, indomethacin, or other non-steroidal anti-inflammatory drugs (as needed medications). Migraine attack, major depressive disorder and analgesic drugs misuse were the main reasons for her hospital admissions.

On November 21, 2015, due to a severe migraine attack she took for the first time in her life codeine $30 \mathrm{mg}$ in combination with acetaminophen $500 \mathrm{mg}$. In that moment, she was prescribed with venlafaxine $225 \mathrm{mg} /$ day $(150 \mathrm{mg}$ in the morning and $75 \mathrm{mg}$ in the afternoon) and diazepam 5 $\mathrm{mg}$ /three times a day (recommended dose). Nevertheless she reported, over the past few mounths that she also took, without any medical supervision, rizatriptan $10 \mathrm{mg}$ as needed use. Her drug intake was basically as needed use and she demonstrated a poor compliance with prescribed medications and doses, but, to the best of our knowledge, she never became dependent to any illicit substances, nether to opiate medications or acohol.

Around 30 to 36 hours after her first codeine intake she contacted a general practitioner reporting symptoms as nervousness, irritability, agitation, mania, confusion, tremor, diaphoresis and nausea. On November 23, she was hospitalized.

The inpatient parameters was: body temperature $37.5^{\circ} \mathrm{C}$; pulse 100 beats/minute; blood pressure $140 / 90$ $\mathrm{mmHg}$ and respiratory rate 21 breaths/minute. Complete blood test (including liver and kidney function tests, creatinine kinase, and serum electrolyte values) were all within normal limits and also the electrocardiogram resulted in the normal range. During the neurological examination it was detected: miotic pupils with decreased light reflex, global hyperreflexia, tremor in both hands, ataxia and incoordination. She had tremors in both hands and anxiety during the interview. No lateralizing neurological signs were observed. The neuropsychological test MODA (Milan Overall Dementia Assessment) was performed and the total score was $87.7 / 100$ (with an age and education adjusted score of $88.5 / 100),{ }^{20)}$ so the test score results in the normal limits. Cranial computed tomography scan were normal and it did not reveal any significant vascular abnormalities.

Venlfaxine and codeine were immediately stopped and endovenous hydration was started with saline solution $0.9 \%$. Diazepam $5 \mathrm{mg}$ was continued orally twice a day for agitation. Approximately 8 hours after the discontinuation of venlafaxine and codeine, the described symptoms disappeared and her hypervigilance improved. Five days later the patient was discharged.

\section{DISCUSSION}

The SS is a predictable response to the increase of serotonin neurotransmission, due to a serotonin drug-drug interaction $^{21)}$ as well as to an individual vulnerability. ${ }^{22)}$ In humans the increased risk of developing the SS is hypothesized by Gelener et al. ${ }^{10)}$ with a possible serotonin transporter polymorphism.

The SS, following concomitant triptan and SSRI (or SNRI) use, is biologically possible. Both of these medications increase serotonin trasmission, therefore, it is predictable that their concomitant use would result in higher serotonin activity. ${ }^{4,8,11)}$ The combination of agitation, diaphoresis, tremor and hyperreflexia in our patient led clinicians to hypothesize a diagnosis of SS, according to Sternbach criteria ${ }^{23)}$ and Hunter criteria. ${ }^{24)}$ Specifically agitation, diaphoresis, tremor and confusion are the Sternbach criteria detected in the patient. Tremor and hyperreflexia are the Hunter criteria observed.

We hypothesize that the development of the SS is connected to the interaction with venlafaxine and rizatriptan 
triggered by codeine.

It is also reasonable to suspect that the symptomatology described was associated with an acute opiate poisoning and/or withdrawal. However, in our opinion the opiate intoxication seems to be less probable because the physicians dind't observe any somnolence, bradycardia, hypotension or bradypnea (inpatient parameters: 100 beats/minute; 140/90 mmHg; 21 breaths/minute). In the same perspective the opiate withdrawal doesn't seem more likely because the patient never stop to ingest codeine, netheir other opiate substances.

As previously mentioned the concomitant use of SSRIs/SNRIs and triptans ${ }^{25}$ could induce the SS: the United States Food and Drug Adminstration Alert ${ }^{26)}$ reporting the potential for life-threatening SS in patients taking SSRIs/SNRIs and triptans concomitantly. This information is based on 27 cases of SS occurring in patients treated with concomitant SSRI or SNRI and triptans.

After a systematic literature review on Medline/ PubMed database we have discovered several cases of SS induced by a combination of antidepressants plus triptans, or antidepressants plus opioids, ${ }^{27)}$ expecially tramadol. ${ }^{28-31)}$ The tramadol involvement in the SS is probably due to the mechanism of action: it binds to mu opioid receptors but also inhibits the monoamine (norepinephrine and 5-HT) reuptake. $^{28,32,33)}$ Contrary to synthetic piperidine (i.e., fentanyl $1^{34)}$ and tramadol ${ }^{28-31)}$ ), codeine, a phenanthrene, does not work as a serotonin uptake inhibitor. ${ }^{33,35,36)}$ Several authors ${ }^{35,36)}$ reported that morphine analogues may potentially increase the intrasynaptic serotonin levels with some unknown mechanisms.

We will propose, as a possible pathophysiological explanation, for the development of SS the cytochromes (CYP2D6 and CYP3A4) involvement, ${ }^{14,36)}$ as also reported by Direk." The genetic individual vulnerability may offer an additional and explanation. ${ }^{9)}$ Addictionally, there are few reportes ${ }^{37,38)}$ indicating that opiates may differentially modulate 5-HT neurotransmission in the central nervous system.

As mentioned in the indroduction, the metabolite morphine, wich has a high affinity for the mu opioid receptor, is primarily responsible for positive and negative effects of codeine tharapy. Altrought the amount of morphine metabolized from codeine is not the same for everybody. This interindividual variability is in part due to the polymorphic cytochrome CYP2D6 enzyme (the mediator of codeine transformation into morphine). As reported by Tao and Auerbach ${ }^{38)}$ morphine infusion into the dorsal raphe nucleus of rats increased extracellular 5-HT in the nu- cleus accumbens. So, one of the possible pathophysiological explanation of this SS case is the iper-biotrasformation of codeine into morphine, mediated in this patient by a high CYP2D6 activity. This mechanism could explain the increase of 5-HT and the consequent trigger role of codeine in our patient, already in therapy with SNRI and triptan.

In conclusion, SS is a severe clinical condition with high mortality. Physicians should be aware of all serotonergic agents prescription.

To the best of our knowledge there are no reports of serotonin toxicity triggered by codeine in a patient in therapy with venlafaxine and rizatriptan was never reported. SS should be kept in mind if patients are in therapy with all substances that may interact with serotonergic drugs through the CYP450 pathway, ${ }^{39)}$ including OTC medications and illicit substances. Moreover meticulous collection of AEs by patients, doctors and pharmaceutical companies should be necessary to investigate in more detail the underlying causes of severe pharmacological interactions.

\section{REFERENCES}

1. Stone JA, Lester CA, Aboneh EA, Phelan CH, Welch LL, Chui MA. A preliminary examination of over-the-counter medication misuse rates in older adults. Res Social Adm Pharm 2017;13:187-192.

2. Fergusson D, Doucette S, Glass KC, Shapiro S, Healy D, Hebert $\mathrm{P}$, et al. Association between suicide attempts and selective serotonin reuptake inhibitors: systematic review of randomised controlled trials. BMJ 2005;330:396.

3. Abbing-Karahagopian V, Huerta C, Souverein PC, de Abajo F, Leufkens HG, Slattery J, et al. Antidepressant prescribing in five European countries: application of common definitions to assess the prevalence, clinical observations, and methodological implications. Eur J Clin Pharmacol 2014; 70:849-857.

4. Blier P, de Montigny C, Chaput Y. A role for the serotonin system in the mechanism of action of antidepressant treatments: preclinical evidence. J Clin Psychiatry 1990;51 Suppl:14-20; discussion 21.

5. Lee $\mathrm{YC}$, Lin $\mathrm{CH}$, Lin MS, Lu Y, Chang CH, Lin JW. Comparison of the effects of serotonin-norepinephrine reuptake inhibitors versus selective serotonin reuptake inhibitors on cerebrovascular events. J Clin Psychiatry 2016; 77:e1-e7.

6. Shin D, Oh YH, Eom CS, Park SM. Use of selective serotonin reuptake inhibitors and risk of stroke: a systematic review and meta-analysis. J Neurol 2014;261:686-695.

7. Vaswani M, Linda FK, Ramesh S. Role of selective serotonin reuptake inhibitors in psychiatric disorders: a comprehensive review. Prog Neuropsychopharmacol Biol Psychiatry 2003;27:85-102.

8. Birmes P, Coppin D, Schmitt L, Lauque D. Serotonin syndrome: a brief review. CMAJ 2003;168:1439-1442.

9. Direk MÇ, Yıldırım V, Güneş S, Bozlu G, Okuyaz Ç. Serotonin syndrome after clomipramine overdose in a child. 
Clin Psychopharmacol Neurosci 2016;14:388-390.

10. Gelener P, Gorgulu U, Kutlu G, Ucler S, Inan LE. Serotonin syndrome due to duloxetine. Clin Neuropharmacol 2011;34: 127-128.

11. Abadie D, Rousseau V, Logerot S, Cottin J, Montastruc JL, Montastruc F. Serotonin syndrome: analysis of cases registered in the french pharmacovigilance database. $J$ Clin Psychopharmacol 2015;35:382-388.

12. Evans RW. The FDA alert on serotonin syndrome with combined use of SSRIs or SNRIs and Triptans: an analysis of the 29 case reports. MedGenMed 2007;9:48.

13. Goadsby PJ, Goldberg J, Silberstein SD. Migraine in pregnancy. BMJ 2008;336:1502-1504.

14. Davanzo R, Bua J, Paloni G, Facchina G. Breastfeeding and migraine drugs. Eur J Clin Pharmacol 2014;70:1313-1324.

15. Madadi P, Amstutz U, Rieder M, Ito S, Fung V, Hwang S, et al. Clinical practice guideline: CYP2D6 genotyping for safe and efficacious codeine therapy. J Popul Ther Clin Pharmacol 2013;20:e369-e396.

16. Nielsen S, Van Hout MC. Over-the-counter codeine-from therapeutic use to dependence, and the grey areas in between. Curr Top Behav Neurosci 2016. doi: 10.1007/ 7854_2015_422. [Epub ahead of print]

17. Seif-Barghi T, Moghadam N, Kobarfard F. Morphine/ codeine ratio, a key in investigating a case of doping. Asian J Sports Med 2015;6:e28798.

18. Foley M, Breindahl T, Hindersson P, Deluca P, Kimergård A. Misuse of 'over-the-counter' codeine analgesics: does formulation play a role? Public Health 2016;130:95-96.

19. Magnus P, Ghavimi B, Coe JW. Access to $7 \beta$-analogs of codeine with mixed $\mu / \delta$ agonist activity via 6,7- $\alpha$-epoxide opening. Bioorg Med Chem Lett 2013;23:4870-4874.

20. Brazzelli M, Capitani E, Della Sala S, Spinnler H, Zuffi M. A neuropsychological instrument adding to the description of patients with suspected cortical dementia: the Milan overall dementia assessment. J Neurol Neurosurg Psychiatry 1994:57:1510-1517.

21. Dvir Y, Smallwood P. Serotonin syndrome: a complex but easily avoidable condition. Gen Hosp Psychiatry 2008;30: 284-287.

22. Fox MA, Jensen CL, Gallagher PS, Murphy DL. Receptor mediation of exaggerated responses to serotonin-enhancing drugs in serotonin transporter (SERT)-deficient mice. Neuropharmacology 2007;53:643-656.

23. Sternbach $\mathrm{H}$. The serotonin syndrome. Am J Psychiatry 1991;148:705-713.

24. Dunkley EJ, Isbister GK, Sibbritt D, Dawson AH, Whyte IM. The Hunter Serotonin Toxicity Criteria: simple and accurate diagnostic decision rules for serotonin toxicity. QJM 2003;96:635-642.

25. Rolan PE. Drug interactions with triptans : which are clinically significant? CNS Drugs 2012;26:949-957.

26. U.S. Food and Drug Administration. FDA ALERT [7/2006]:
Potentially life-threatening serotonin syndrome with combined use of SSRIs or SNRIS and Triptan medications [Internet]. Rockville, MD.: U.S. FDA, 2013 Aug 14 [cited 2016 Feb 12]. Available from: http://www.fda.gov/Drugs/ DrugSafety/PostmarketDrugSafetyInformationforPatientsan dProviders/DrugSafetyInformationforHeathcareProfessional s/ucm085845.htm.

27. Greenier E, Lukyanova V, Reede L. Serotonin syndrome: fentanyl and selective serotonin reuptake inhibitor interactions. AANA J 2014;82:340-345.

28. Shakoor M, Ayub S, Ahad A, Ayub Z. Transient serotonin syndrome caused by concurrent use of tramadol and selective serotonin reuptake inhibitor. Am J Case Rep 2014; 15:562-564.

29. Albiñana Pérez MS, Cea Pereira L, Bilbao Salcedo J, Rodríguez Penín I. [Possible serotonin syndrome associated with administration of venlafaxine and tramadol]. Farm Hosp 2012;36:548. Spanish.

30. Sauget D, Franco PS, Amaniou M, Mazere J, Dantoine T. [Possible serotonergic syndrome caused by combination of tramadol and sertraline in an elderly woman]. Therapie 2002;57:309-310. French.

31. Takeshita J, Litzinger MH. Serotonin syndrome associated with tramadol. Prim Care Companion J Clin Psychiatry 2009;11:273.

32. Mittino D, Mula M, Monaco F. Serotonin syndrome associated with tramadol-sertraline coadministration. Clin Neuropharmacol 2004;27:150-151.

33. Codd EE, Shank RP, Schupsky JJ, Raffa RB. Serotonin and norepinephrine uptake inhibiting activity of centrally acting analgesics: structural determinants and role in antinociception. J Pharmacol Exp Ther 1995;274:1263-1270.

34. Ailawadhi S, Sung KW, Carlson LA, Baer MR. Serotonin syndrome caused by interaction between citalopram and fentanyl. J Clin Pharm Ther 2007;32:199-202.

35. Rastogi R, Swarm RA, Patel TA. Case scenario: opioid association with serotonin syndrome: implications to the practitioners. Anesthesiology 2011;115:1291-1298.

36. Gillman PK. Monoamine oxidase inhibitors, opioid analgesics and serotonin toxicity. Br J Anaesth 2005;95:434441.

37. Karunatilake H, Buckley NA. Serotonin syndrome induced by fluvoxamine and oxycodone. Ann Pharmacother 2006; 40:155-157.

38. Tao R, Auerbach SB. Opioid receptor subtypes differentially modulate serotonin efflux in the rat central nervous system. $J$ Pharmacol Exp Ther 2002;303:549-556.

39. Pilgrim JL, Gerostamoulos D, Drummer OH. Review: Pharmacogenetic aspects of the effect of cytochrome P450 polymorphisms on serotonergic drug metabolism, response, interactions, and adverse effects. Forensic Sci Med Pathol 2011;7:162-184. 